\title{
Electrical Impedance in the Subicular Area of Rats during Paradoxical Sleep
}

\author{
JaMes B. Ranck, JR. ${ }^{1}$ \\ Department of Physiology, The University of Michigan, Ann Arbor, Michigan
}

Received August 3, 1966

A four-electrode method of chronically measuring impedance of less than $1 \mathrm{~mm}^{3}$ parts of brain in unrestrained rats is described. The method is usually good from about 1.5 to $3,000 \mathrm{cycle} / \mathrm{sec}$ for the absolute value of phase angle, and relative changes in magnitude of impedance can be measured from about 1.5 to 10,000 cycle/sec. Testing current of $2 \times 10^{-8} \mathrm{amp}$ is used, which produces a signal of about $20 \mu \mathrm{v}$. Stability for hours and days is sometimes, but not always, achieved. In the subicular area, there is invariably an increase in magnitude of impedance of up to $25 \%$ during paradoxical sleep. In the same animal, this increase is the same during all episodes of paradoxical sleep lasting longer than $1 \mathrm{~min}$, and is the same at 32 to $10,000 \mathrm{cycle} / \mathrm{sec}$. The phase angle at 100 to $1,000 \mathrm{cycle} / \mathrm{sec}$ becomes 1 or $2 \mathrm{deg}$ more negative during paradoxical sleep. There are no changes or very small changes in Ammon's horn and the fascia dentata. Because these data are fairly complete, the mechanisms which might possibly be responsible for these impedance changes can be stated specifically in semiquantitative form. Among several possibilities, a single one cannot be chosen on the basis of these impedance data alone, but from other considerations, a decreased size of interstitial space seems by far the most likely.

\section{Introduction}

Since 1953 there have been many reports of changes in the electrical impedance of the brain associated with various functional states. It has been difficult to interpret many of these results for several reasons: inadequacies of the methods used, incompleteness of data, and uncertainty about pathways of current flow in the brain. These difficulties are compounded because many of the possible interpretations involve mechanisms whose occurrences are merely hypothesized or poorly understood. Evidence from impedance measurements is sufficiently indirect so that it is unwise to push its implications very far. On the other hand, these very suggestions of as yet unappreciated mechanisms are those which make impedance measurements of the brain intriguing.

1 This study was supported by grant NIH NB-04352 from The National Institutes of Health. I thank Constance Jones, who made electrodes and operated and ran many rats, and Frode Maaseidvaag and Kenneth Burkhalter for help in the design and construction of electronic equipment. 
In earlier papers (10-13), some headway into interpreting impedance data was made by measuring absolute value of magnitude and phase angle of impedance at a variety of frequencies in the tradition of Cole. In this paper, these methods are extended to long-term measurement with electrodes chronically implanted in the brain. Four main features will emerge from this paper.

$i$. The method itself is described in some detail and is shown to have some special difficulties and to be subject to many errors. By and large, these are controllable by methods explained. The method has several advantages over previously used methods and allows fairly complete data to be collected so that some semiquantitative interpretation is possible.

ii. The impedance change in the subicular areas during paradoxical sleep is shown to be the largest change seen in the brain under normal conditions.

iii. The possible causes of this impedance change can be delineated, although this impedance method does not allow us to distinguish between these possibilities. On the basis of other considerations, it seems very likely that the change is due to an increased resistance of the interstitial space of the brain.

$i v$. So far as I know, this is the first instance of a change in the subicular area, not occurring in Ammon's horn, associated with normal functioning. Its significance for paradoxical sleep is not clear.

\section{Methods}

All previously reported data on the measurement of impedance in the brain of animals in chronic experiments were obtained by a two-electrode method (the same electrodes were used to pass current and to record voltage difference). The four-electrode method (two electrodes pass current, and two other electrodes record the voltage difference) has previously been used in acute preparations $(8,10,12,13)$. The four-electrode method has the advantage that the effects of electrode polarization, the impedance at the electrode tissue interface, and the tissue which has been damaged by the electrode, yet electrically is in series with the electrodes, can largely be ignored. This advantage has four implications, as compared with the two-electrode method, for in vivo use: $i$. The absolute value of the phase angle of the impedance can be measured only by a four-electrode method. Even at high frequencies, the electrode polarization introduces sizable phase angles in the two-electrode method. ii. Frequencies below about $1,000 \mathrm{cycle} / \mathrm{sec}$ can be utilized with a four-electrode method. There is indeed no lower limit to the frequencies which can be used. iii. There is little question that changes in impedance with a fourelectrode method are due to the tissue and not to the electrode tissue interface, and less chance that changes are due to change in the damaged tissue. $i v$. The impedance changes measured are not decreased because of the impedance of some constant electrode tissue interface or damaged tissue in series. 
However, the four-electrode method is subject to some special errors. It will be convenient to discuss three types of these errors before describing the details of the method.

\section{A. Linear current configuration}

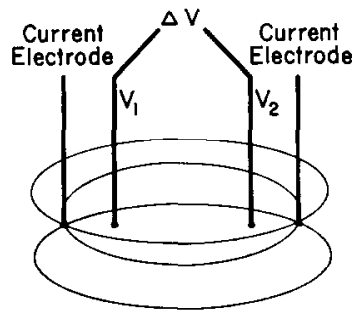

B. Radial current configuration

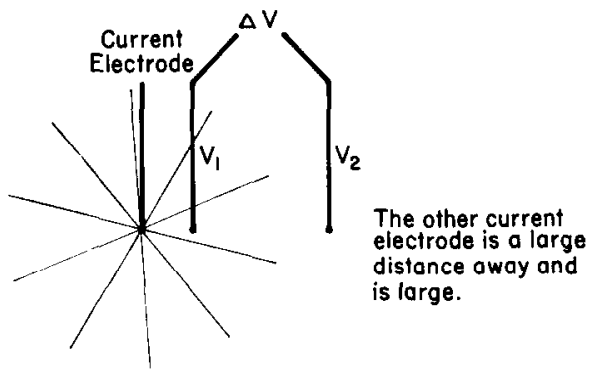

C.

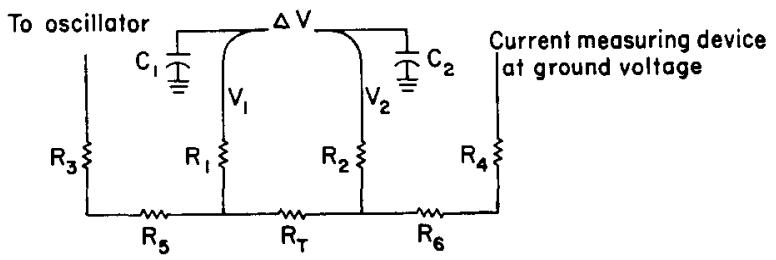

Fig. 1. A and B: Electrode configurations used. The fine lines are lines of current flow. The thick lines are electrodes, insulated except at the tip. C: An equivalent circuit of the electrodes in tissue. This equivalent circuit is surely very incomplete. The resistances are not necessarily linear, and there should also be capacitative and voltage generating elements to express polarization.

Figure $1 \mathrm{~A}$ and $\mathrm{B}$ are diagrams of the electrode configuration used. Figure $1 \mathrm{C}$ is an electrical analog of these situations in which $R_{1}, R_{2}, R_{3}$, and $R_{4}$ are the electrode resistances, and $R_{5}, R_{6}$, and $R_{T}$ are equivalent tissue resistances. $R_{T}$ is the resistance we are trying to measure. If there is a leakage resistance from one of the voltage-recording electrodes to ground 100 times the resistance of the electrode (say, $100 \mathrm{R}_{1}$ ), there will be a $1 \%$ error in 
the voltage recorded at that electrode. However, the ratio of common mode signal to differential signal (the differential signal being the desired one) is $\left(\mathrm{R}_{4}+\mathrm{R}_{6}\right) / \mathrm{R}_{\mathrm{T}}$. Therefore, to obtain the desired accuracy of less than $1 \%$ error, the leakage resistance from a recording electrode to ground must be greater than $100 R_{1}\left(R_{t}+R_{6}\right) / R_{T} ; R_{T}$ is of the order of 500 ohms. For the linear current electrode configuration $\left(R_{4}+R_{6}\right)$ is about the same as $R_{1}$, so the leakage resistance must be greater than $R_{1}{ }^{2} / 5$. Values of $R_{1}$ are:

\begin{tabular}{cccc}
$\begin{array}{c}\text { Frequency } \\
\text { (cycle/sec) }\end{array}$ & $\begin{array}{c}\text { Range of } R_{1} \\
(\mathbf{K})\end{array}$ & $\begin{array}{c}\text { Typical } R_{1} \\
(\mathbf{K})\end{array}$ & $\begin{array}{c}\left(\mathrm{R}_{1} 2 / 5\right) \\
\text { for } \\
\text { Typical } \mathrm{R}_{\mathbf{1}}\end{array}$ \\
\hline 1.5 & $60-900$ & 300 & $18 \times 10^{9}$ \\
32 & $20-160$ & 60 & $7 \times 10^{8}$ \\
1,000 & $10-70$ & 20 & $8 \times 10^{7}$
\end{tabular}

Leakage resistances from a voltage-recording electrode to the wire from the oscillator or to the current-recording electrode will give similar errors. Maintaining resistances of this magnitude on the head of an animal is difficult. For the radial current configuration $R_{4}$ is less, and this problem is also less, but still is one which must be faced.

Consider again Fig. 1C. If $1 /\left(\omega C_{2}\right)>10 R_{2}$, where $\omega$ is $2 \pi$ times the frequency in cycle/sec, and if $\mathrm{C}_{1} \ll \mathrm{C}_{2}$, then the error introduced in the phase angle of impedance will be an angle of approximately $\arctan \left(\omega \mathrm{R}_{2} \mathrm{C}_{2}\left(\mathrm{R}_{6}+\right.\right.$ $\left.R_{4}\right) / R_{T}$ ). The percentage error introduced in magnitude of impedance will be approximately $\left[1-\left(1+\omega^{2} R_{2}^{2} C_{2}{ }^{2}\right)^{1 / 2}\right]\left(R_{6}+R_{4}\right) / R_{T}$. At 1,000 cycle/ sec, $\left(R_{6}+R_{4}\right)$ and $R_{2}$ are about 20,000 ohms, and $R_{T}$ is of the order of 500 ohms. If $\mathrm{C}_{2}=30 \mu \mu \mathrm{f}$ (which it does) at $1,000 \mathrm{cycle} / \mathrm{sec}$, the phase angle error will be $8.6 \mathrm{deg}$. The error in magnitude will be $0.028 \%$. At 10,000 cycle/sec, the phase angle error is $56 \mathrm{deg}$, and the error in magnitude $2.8 \%$. If $\mathrm{R}_{2}$ were $100,000 \mathrm{ohms}$ (and some are) at $10,000 \mathrm{cycle} / \mathrm{sec}$, the error in magnitude would be $70 \%$. The effect of increasing $\mathrm{C}_{1}$ into the range of $\mathrm{C}_{2}$ is to decrease, and at one point to eliminate, these errors. However, the exact result will depend on the electrode resistance which is the factor we had hoped to avoid by the four-electrode method. In the radial configuration, these errors are much less.

The recording electrodes are fairly thick, compared to the interelectrode distances (Fig. 2). The difference in potential between one side and the other side of the electrode is not negligible, compared to the signal. The potential actually recorded by the electrode will be intermediate, the exact value depending on the details of the electrode tissue interfacial resistance, which can be assumed to change with frequency and perhaps over a period of time. To decrease this error, the exposed electrode tips could be smaller (thereby increasing electrode resistance) or the interelectrode distances increased (thereby decreasing the localization). 
Adey, Kado and Didio (1) used a phase-locked sampling method to measure impedance of tissue in a case in which the signal is much less than the noise (mostly the spontaneous activity of the brain). This allows very small currents to be passed through the brain. Indeed, to be sure that the testing current is not itself changing the impedance, currents must be so small that some method of pulling the signal out of a much larger noise must be used. The lock-in-amplifier used in this study is different hardware from that

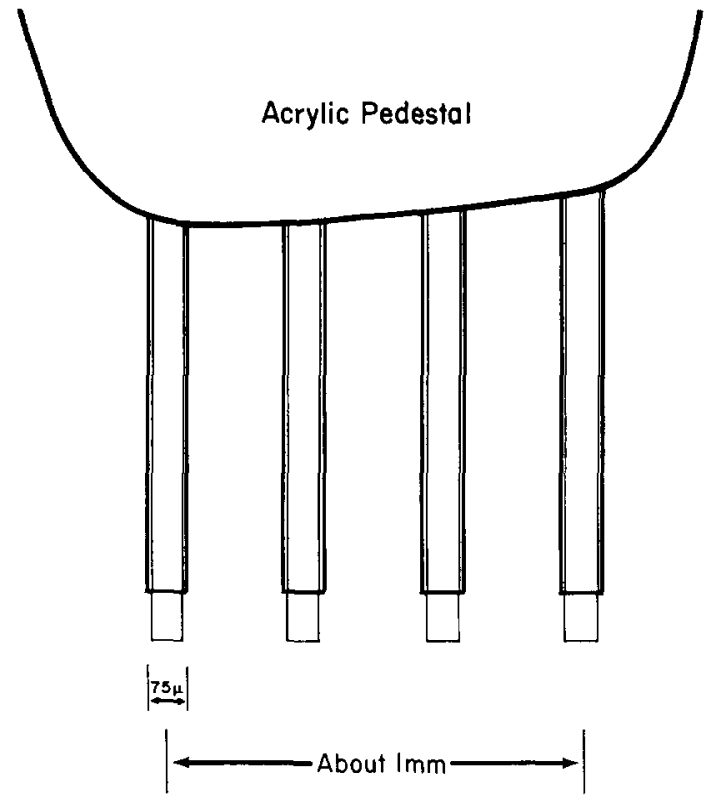

FIG. 2. The end of the complete electrode. The electrodes are insulated to within about $100 \mu$ of the end. Note that by far the largest part of the current flows through tissue which is undamaged, and the major tissue damage near the large pedestal should effect current flow very little. In the linear configuration, the impedance of tissue about $300 \times 500 \times 500 \mu$ is being measured. In the radial configuration, the impedance of spherical shell of tissue with inside radius of about 200 to $500 \mu$, and outer radius of about 600 to $1,200 \mu$, is being measured.

used in the sampling method of Adey, Kado and Didio. However, both are cross correlation methods, and resolution of signal from noise in theory is exactly the same in both methods. The signal is of the order of $20 \mu \mathrm{v}$, and the spontaneous activity is of the order of $300 \mu \mathrm{v}$.

In measuring impedance in rats, in calibrating equipment, and in measuring electrode resistances, about $2.5 \times 10^{-8} \mathrm{amp}$ of peak sinusoidal current is passed. The surface of each electrode is about $25,000 \mu^{2}$, so the current density is about $10^{-12} \mathrm{amp} / \mu^{2}$ or $0.1 \mathrm{mamp} / \mathrm{cm}^{2}$. Adey, Kado and Didio (1) passed less than $5 \times 10^{-8}$ amp through larger electrodes with a current dens- 
ity of about $10^{-1 . \prime} \mathrm{amp} / \mu^{2}$. In this four-electrode method, the current density through the tissue in which the impedance is actually measured is also of the order of $10^{-13} \mathrm{amp} / \mu^{2}$, and the voltage differences are about $20 \mu \mathrm{v}$. With Adey, Kado and Didio, I assume this does not stimulate. One rat with electrodes in the subiculum was run for 6 hours at $8 \times 10^{-9} \mathrm{amp}$. The results were the same as those at three times this current, including the changes during paradoxical sleep.

Impedance was measured in one rat while current was varied from $5 \times 10^{-9}$ to $5 \times 10^{-8} \mathrm{amp}$. The same value of impedance was obtained in all cases. This shows that the device really is averaging out the signal independent of the spontaneous activity of the brain, for the signal is varied tenfold while the spontaneous activity remains constant. This test also demonstrates that the impedance is independent of current strength.

Figure 3 is a diagram of the electrical arrangement. ${ }^{2}$ The variable phase shifter, the oscillator, tuned amplifier, and lock-in-amplifier are all part of the same device, a model JB-5 lock-in-amplifier from the Princeton Applied Research Corporation. The impedance matching devices have a gain of 0.99 , grid current of less than $10^{-13} \mathrm{amp}$, an input resistance of greater than $10^{9} \mathrm{ohms}$, and an input capacity of $30 \mu \mu \mathrm{f}$. The differential amplifier with impedancematching inputs has a rejection ratio of better than 1,000:1 over the frequency range used. The current-measuring device is an operational amplifier utilized as a "transresistor," which yields an output voltage proportional to the input current. The effective input impedance of this circuit is on the order of milliohms.

The output of the phase shifter is the reference voltage, and the output of the tuned amplifier is the signal voltage of the lock-in-amplifier. The output of the lock-in-amplifier is a d-c voltage equal to $A K \cos \theta$, where $A$ is a constant of the system, $K$ is the amplitude of the signal input, and $\theta$ is the phase angle between the signal and reference voltages. That part of the signal which occurs at some frequency other than the reference frequency, or with a varying phase angle (i.e., the noise), is averaged out. The time constant of averaging can be varied, but is usually $1 \mathrm{sec}$ at $1,000 \mathrm{cycle} / \mathrm{sec}$ and $10 \mathrm{sec}$ at 32 cycle $/ \mathrm{sec}$. The output is independent of the amplitude of the reference input. at least for changes of up to $10 \%$. The output of the rectified and integrated reference voltage is a d-c voltage of opposite polarity of the output of the lock-in-amplifier.

Potentiometers I and II are set so that there is zero output. Note that if $A=B=1$, then $\ln d A=d A$ and $\ln (d A / d B)=\ln d A-\ln B=d A-d B$ $=d A d B$. Therefore, a subtractor of equal signals is an accurate analog divider. Indeed, changes of up to $20 \%$ from the zero output point are re-

2 Details of the electronic circuits are available on request. 
corded with only a $10 \%$ error. Changes of current of up to $15 \%$ do not change the output of this system by more than $0.5 \%$. During runs, the current passed does not change more than $1 \%$.

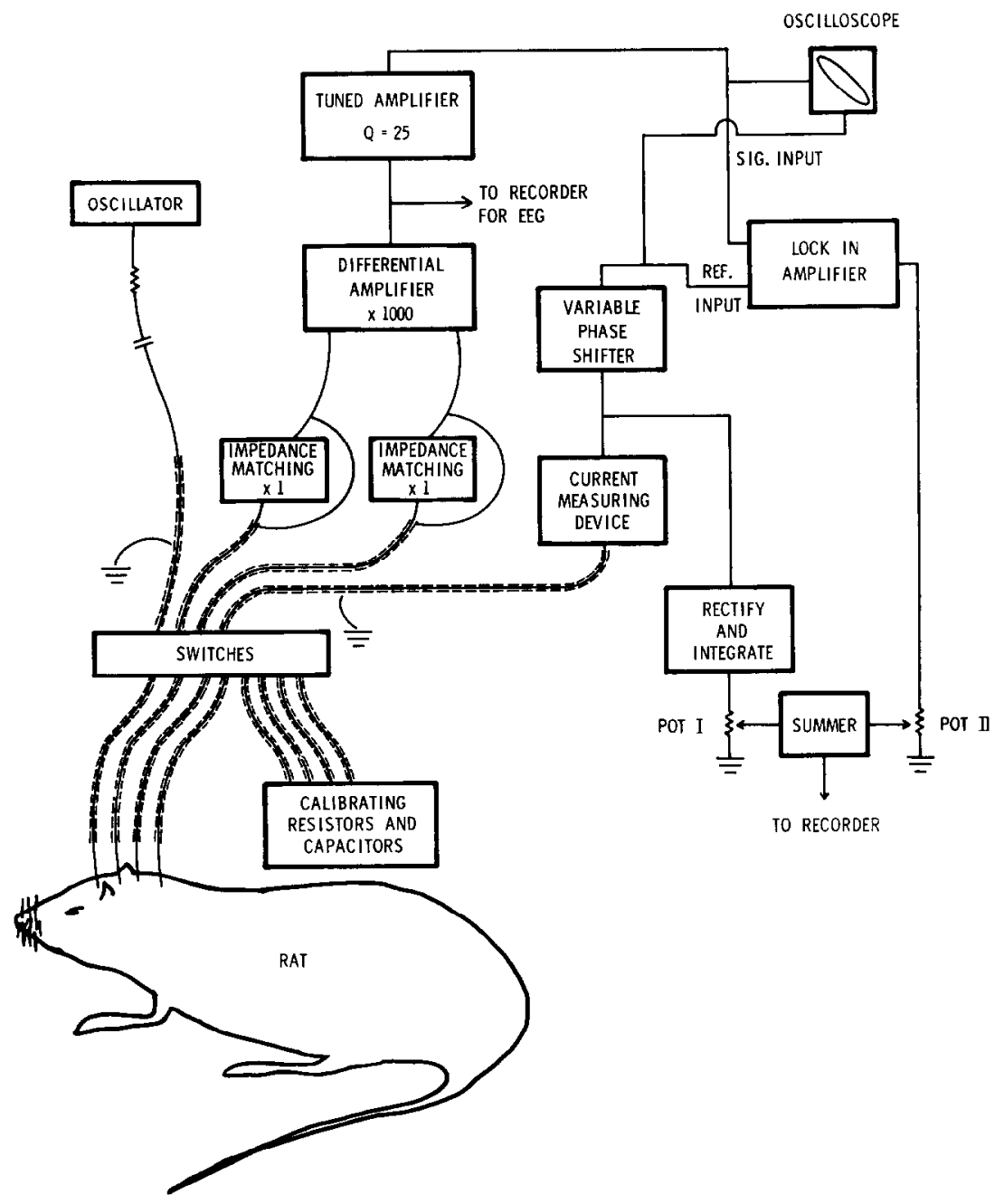

FIG. 3. Diagram of the electrical arrangement. See text for explanation.

The magnitude of the impedance was measured by adjusting the phase shifter of the reference in the calibration mode with a resistor as the test impedance so that there was no phase angle $(<1 \mathrm{deg})$ between the signal and reference voltages. Since all phase angles in the tissue were less than $10 \mathrm{deg}$, and $\cos 10 \mathrm{deg}=0.985$, the voltage output of the system when running on tissue is effectively the magnitude of impedance. 
The phase angle was measured by shifting the reference voltage $90 \mathrm{deg}$ from the signal in the calibration mode. For a constant amplitude of the testing current, the output of the lock-in-amplifier is proportional to the magnitude of the impedance times sin of the phase angle of the impedance being tested.

The electrodes were $75-\mu$ wires of $92 \%$ platinum, $8 \%$ tungsten. They were insulated with four to six coats of Formvar. Four wires were fixed in a mold of dental acrylic, with acrylic covering the wires to within about $1 \mathrm{~mm}$ of the tips. The whole thing was insulated with three coats of Epoxylite. The final 75-100 $\mu$ of insulation of the electrode was scraped off and bare wire was platinized. The four tips were in a straight line, about equally spaced. The entire distance over which the electrode tips were spaced was 0.1 to $1.5 \mathrm{~mm}$. Therefore, the distance from one electrode tip to an adjacent electrode tip was 0.2 to $0.4 \mathrm{~mm}$. Figure 2 is a scale drawing of the end of the complete electrode. Let us call each wire an electrode, and the four electrodes in the acrylic the "complete electrode." Errors due to leakage between electrodes or to ground will have a different phase from the signal. Therefore, it is convenient to measure leakage errors by measuring phase angles at $1.5 \mathrm{cycle} / \mathrm{sec}$. The complete electrodes were tested in $0.2 \% \mathrm{NaCl}$ at $1.5 \mathrm{cycle} / \mathrm{sec}$. All possible combinations of the four electrodes giving the radial current configuration (12) and one combination of the linear current configuration were used. If there was any discernible phase angle (greater than $2 \mathrm{deg}$ ), the complete electrode was not used.

Sprague-Dawley rats weighing between 250 and $500 \mathrm{~g}$ each were used. The complete electrode was placed stereotaxically in an anesthetized rat, with the tips of the electrodes horizontal and in an anterior-posterior line. A platinized platinum wire was inserted into extracranial tissue. This served as the current electrode a large distance from the other which was used in the radial current configuration.

When impedance was measured, the top of the complete electrode in the rat was cleaned with $70 \%$ alcohol. The electrode was connected to wires leading to the electronic apparatus and electrical tape was wrapped around the electrode holder and complete electrode so the rat could not touch the wires or the top of the electrode. The rat was placed in a shielded cage containing a water bottle and food.

The phase angle of the impedance was observed on the oscilloscope at 1.5 cycle/sec at the electrode configurations to be used. Often the phase angle would decrease over the first 10-20 min, presumably as the water and alcohol used to wash the top of the complete electrode evaporated. The animal was run only if the phase angle was less than $10 \mathrm{deg}$. The voltage due to the signal current was read in the presence of spontaneous activity of the brain. The tuned amplifier eliminated some of this noise, but it was often possible 
to say no more than that the phase angle was less than $10 \mathrm{deg}$. The phase angle of the impedance at $1.5 \mathrm{cycle} / \mathrm{sec}$ was also measured at the end of a run, at which time it was also required to be less than $10 \mathrm{deg}$.

Rats were usually run at one radial current and one linear current configuration at 32 and 1,000 and $3,200 \mathrm{cycle} / \mathrm{sec}$. Frequencies of less than 32 cycle/sec were not used routinely for three reasons. $i$. The possibility of errors due to leakage is increased as the frequency is decreased. $i i$. The lower the frequency, the longer the averaging time, because it takes longer to collect the sample, and the slower the response of the system. iii. The major frequencies of the spontaneous activity of brain are between about 4 and 20 cycle/sec. In this range, then, the "noise" cannot be filtered out from the signal as effectively, and much longer averaging times are required.

Frequencies higher than about $3,000 \mathrm{cycle} / \mathrm{sec}$ were not used often because of danger of error from input capacities. Indeed, at frequencies as low as $3,000 \mathrm{cycle} / \mathrm{sec}$ in some animals, there were positive phase angles of impedance, which, undoubtedly, is an error. Relative changes are no doubt valid at frequencies somewhat higher than this, but caution is necessary.

\section{Results}

The electrodes were all placed in the dorsal part of the hippocampal formation, 3 to $4 \mathrm{~mm}$ lateral to the midline (Fig. 4). The results of seven animals are reported in this paper; four with electrodes in the subicular area, two in Ammon's horn, and one in the fascia dentata and its hilus.

Figure 5 shows the results of runs over a period of 3 months. In the sub-
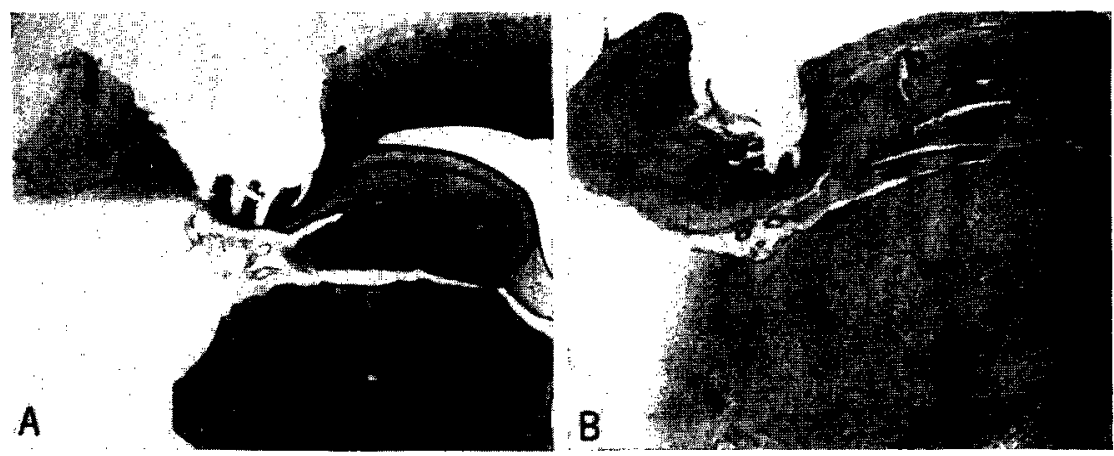

FIG. 4. Photomicrographs of electrode placements in the subicular area. Parasagittal frozen sections with Kluver-Barberra stain. The rostral direction is to the right. The tracts from single electrodes can be seen. All other placements in the subicular area are in the same location as these two, just under the dorsal hippocampal commissure. Those in Ammon's horn and fascia dentata are just anterior to this location. The neocortex was lost in histological preparation in B. In no cases do the electrode tips go all the way through the subicular area. 
icular area, this is about the greatest stability obtained. In other areas, the magnitude of impedance changed by a factor of up to two (but usually within $20 \%$ ) over periods of as short as a few days. Figure 6 shows the magnitude of impedance of a radial configuration of electrodes in the subicular area run

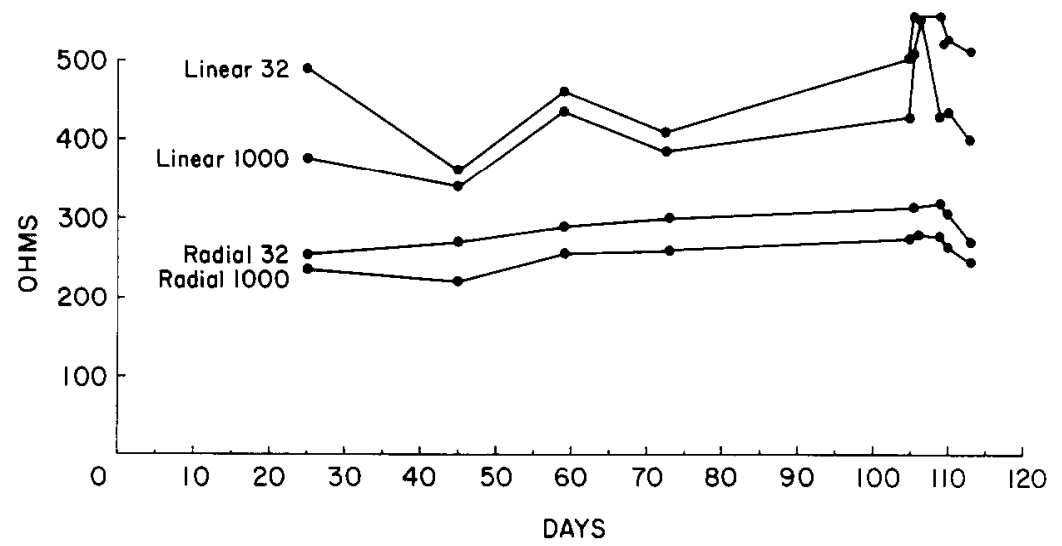

Fig. 5. The variation in magnitude of impedance in subicular area with time, measured from the day of operation. "Linear 32 " refers to a linear current configuration at $32 \mathrm{cycle} / \mathrm{sec}$, etc.
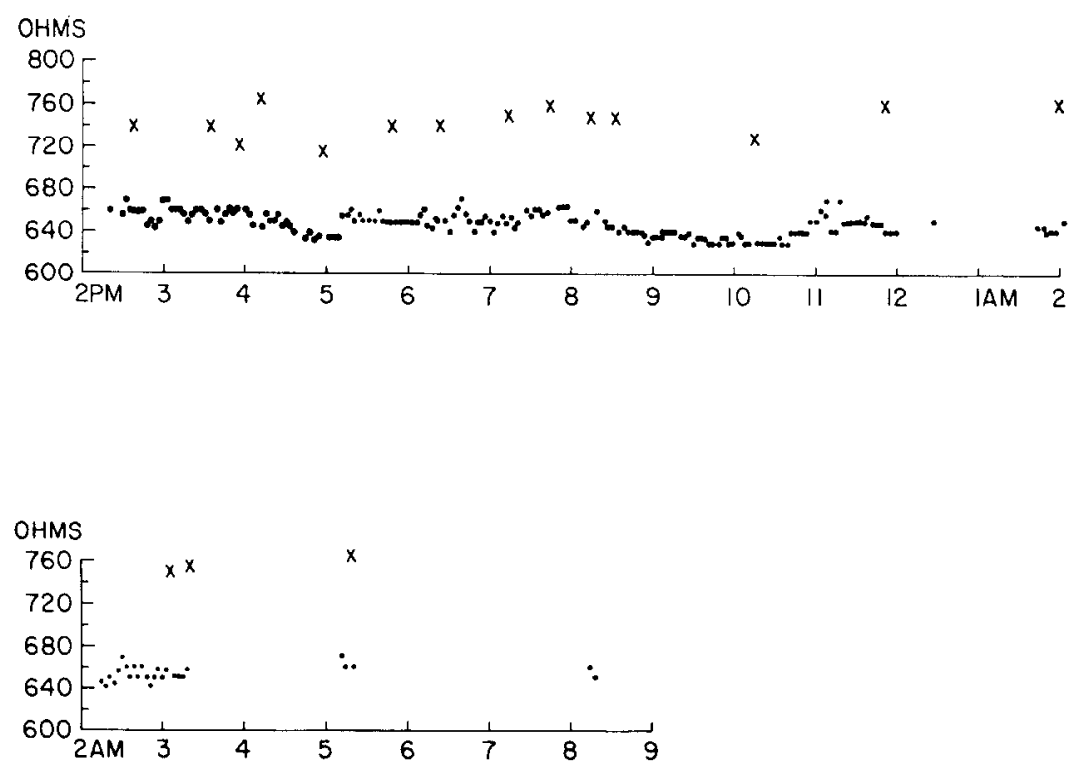

Fig. 6. Magnitude of impedance at $1,000 \mathrm{cycle} / \mathrm{sec}$, radial current configuration, run over an 18-hour period in the subicular area. Each dot represents an average, taken by eye, for a 3-min period. The " $x$ 's" are the increases in magnitude of impedance during episodes of paradoxical sleep. This is from the same rat as in Fig. 7 and 9. 
for 18 hours. The maximal variation was $3 \%$ from the mean. Two other rats were also run almost continuously for about 24 hours with the linear configuration. In one of these, the baseline varied from 430 to $550 \mathrm{ohms}$; in the other, from 170 to $380 \mathrm{ohms}$. Stability over periods of hours or days seems to be better with use of the radial configuration than with the linear, as would be expected from the discussion of errors. However, even though a good stability could be maintained in some animals, this has not been uniformly achieved. Some of these changes in baseline may be biological, whereas some are surely errors. Because of this uncertainty, changes over periods of longer than 1 hour will not be reported in this paper. Those changes that are reported occurred invariably, no matter how stable the magnitude of impedance.

The electrodes were tested at $1.5 \mathrm{cycle} / \mathrm{sec}$ in a $\mathrm{NaCl}$ solution with a resistivity of $258 \mathrm{ohm}-\mathrm{cm}$. The results at each configuration were then compared with the results in the brain to give the resistivity of the brain. These results must be considered as very crude approximations because of the following. $i$. There is lack of stability with time in some cases. $i i$. The relative positions of the electrode tips to each other may change during insertion into the brain. iii. It has not been determined that the tissue is isotropic. All placements in fascia dentata, Ammon's horn, or subicular area gave values of about $250 \mathrm{ohm}-\mathrm{cm}$. I think it likely that the real value does not differ by more than a factor of two from this. Because of the uncertainties in the absolute values, the results of magnitude of impedance will be given in raw equivalent ohms; this cannot be directly correlated with specific impedance. (The phase angle is a good absolute value, except for any error due to anisotrophy; 10, p. 150.)

Because of these uncertainties in the absolute value of the impedance, and because errors which do exist may well be different at different frequencies, no complete phase plane data will be given. However, the frequency dependence and phase angles of impedance were similar to that of rabbit cerebral neocortex. The magnitude of impedance was almost always less at 1,000 or $3,200 \mathrm{cycle} / \mathrm{sec}$ than at 10 and $32 \mathrm{cycle} / \mathrm{sec}$. For frequencies less than $3,000 \mathrm{cycle} / \mathrm{sec}$, phase angles were always negative (usually less than 5 $\mathrm{deg}$ ) and the largest phase angles were at 32 or $100 \mathrm{cycle} / \mathrm{sec}$. But the data are not good enough to warrant being given more quantitatively.

The major finding was associated with episodes of paradoxical sleep. Paradoxical sleep was identified by the criteria of observation of the rat and the spontaneous activity recorded from the part of brain being studied. Our observations are in general agreement with those of Roldan, Weiss and Fifkova (14). Invariably, there was twitching of vibrissae and, often, of the nose and ears, and there was a theta rhythm of about $6 / \mathrm{sec}$ during episodes of paradoxical sleep. The episode invariably ended abruptly with the rat making a 
sudden movement. Out of the more than 200 episodes of paradoxical sleep observed, in fewer than 5 cases was there any question as to placement in that catcgory, cven though more complete recording was not uscd. All ambiguous episodes were of less than $1 \mathrm{~min}$ in duration.
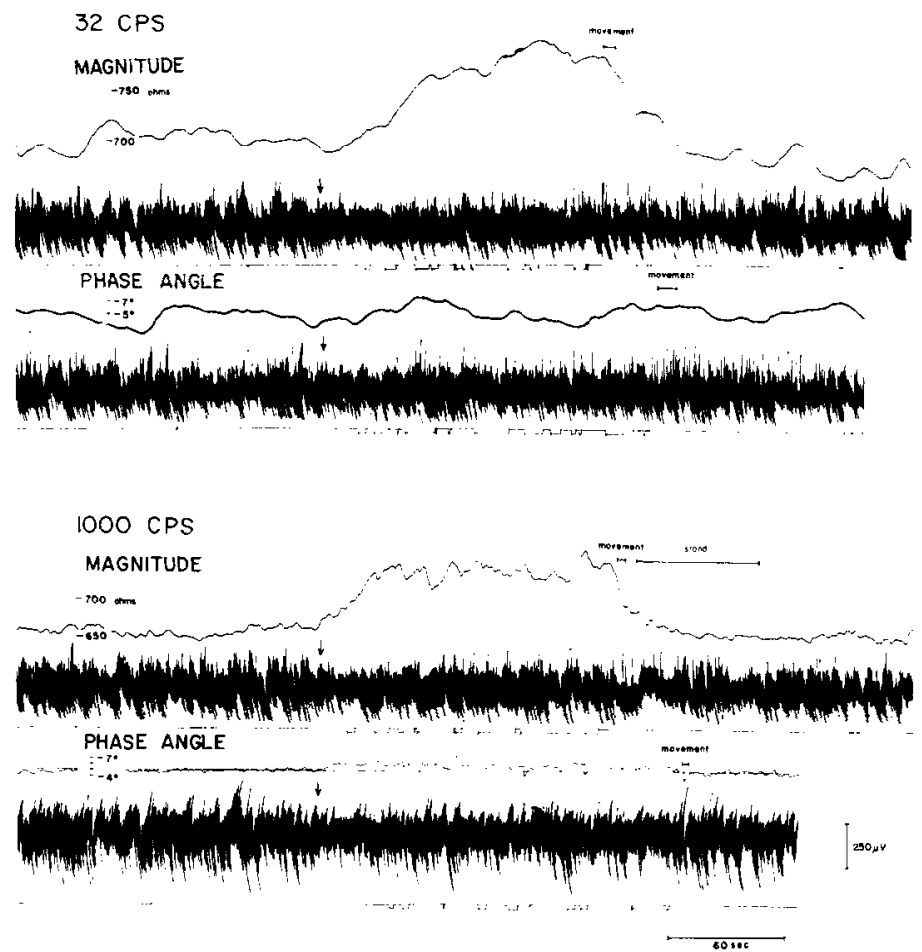

Fig. 7. A composite of impedance changes in the subicular area during paradoxical sleep. Each component of the impedance is from a separate episode of paradoxical sleep, but all are from the same rat. This is raw data. In each run the top line is that component of impedance as labeled. The second line is the spontaneous electrical activity. The bottom line indicates twitching of the vibrissae when it goes down. The arrows indicate the beginning of the theta rhythm. The voltage calibration in the lower right corner applies to the spontaneous electrical activity. In the phase angle for 1,000 cycle/sec a baseline at $-5 \mathrm{deg}$ has been added. Unless otherwise stated that rat is lying down quietly with his head down and his eyes closed.

During paradoxical sleep, the magnitude of the impedance in the subicular area invariably increased (Fig. 7). It can take up to 1 min before the maximum is reached. Because of the variation in magnitude of impedance during paradoxical sleep and the differing response time at different testing frequencies, there is not a simple way of measuring the increase that will be comparable at different frequencies. Somewhat arbitrarily, the increase was 
the maximal value which the record maintained or surpassed for at least 10 sec. The percentage changes of magnitude of impedance during episodes of paradoxical sleep which lasted at least 1 min are listed in Table 1. All technically acceptable runs are included. At a given frequency and configuration, the change is remarkably constant. The one exception to this is the linear configuration of No. 35 at $1,000 \mathrm{cycle} / \mathrm{sec}$. The two grouped runs, which are larger than the rest, were run 7 weeks after the others, and the animal was sick at these later times. Although these data are included for completeness, I conclude that it was the long delay or the illness of the rat that caused the change, and that, with these qualifications, the change during parodoxical sleep is reproducible. Figure 6 shows the increase in magnitude of impedance at $1,000 \mathrm{cycle} / \mathrm{sec}$ many times in 1 day.

Another important result shown in Table 1 is that for a given rat and a given electrode configuration, the changes are the same at all frequencies within the limits of error of the measurement.

Changes in phase angle of impedance in the subicular area during paradoxical sleep were measured in two animals. In one rat, no changes were seen. In another rat, the phase became 1-2 deg more negative at 100 and $1,000 \mathrm{cycle} / \mathrm{sec}$; at $32 \mathrm{cycle} / \mathrm{sec}$, if there was any change, it was less than 2 deg.

Figure 7 is a composite of the changes in impedance in the subicular area during paradoxical sleep showing the magnitude and phase angle at 32 and $1,000 \mathrm{cycle} / \mathrm{sec}$. Each of these four measurements was from a separate episode of paradoxical sleep, but the changes are constant enough so that they can be compared with each other.

Those episodes of paradoxical sleep which lasted less than a minute and, therefore, may not have had as large a maximum as a longer episode, nevertheless had changes in impedance which appeared similar to the early stages of a longer episode (Fig. 9A).

At 1,000 cycle/sec and higher, a gradual increase in magnitude of impedance was often (but not always) seen for as long as $1 \mathrm{~min}$ before the theta rhythm began. A small increase is shown in Fig. 7, although Fig. 8A and B show it better. Figure $8 \mathrm{~A}$ is recorded at a faster paper speed, and the abrupt beginning of the theta rhythm is clearly seen. The magnitude of impedance began to increase even before this. Figure $8 \mathrm{~B}$ shows the results from another animal where the transition of the theta rhythm is clear even at the slow paper speed. When the theta rhythm begins, the magnitude of impedance continues to increase, usually with an increased rate. The increases to maxima are often associated with twitching of vibrissae of a rat, which usually does not occur until after 10 to $30 \mathrm{sec}$ of theta rhythm. Subsequent increases of magnitude of impedance during the episode of paradoxical sleep often occurred during episodes of twitching of vibrissae. 


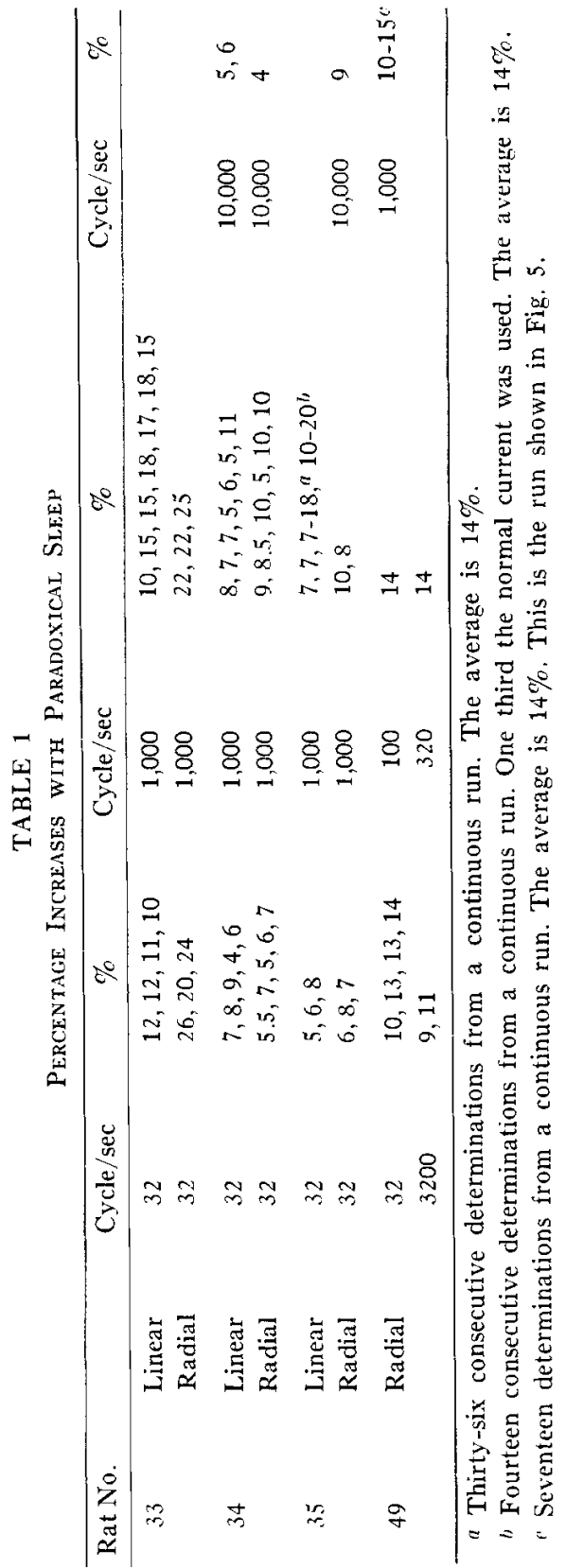


Episodes of paradoxical sleep were invariably terminated by an abrupt movement of the whole body of the rat. Within $1 \mathrm{sec}$ of this movement, the theta rhythm disappeared and the magnitude of impedance began to decrease. The magnitude of impedance returned to the previous level within $1 \mathrm{~min}$. This abrupt decrease in magnitude of impedance associated with the

MAGNITUDE

A $1000 \mathrm{CPS}$

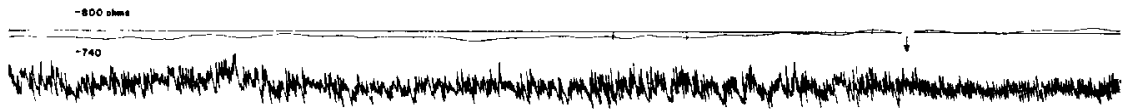

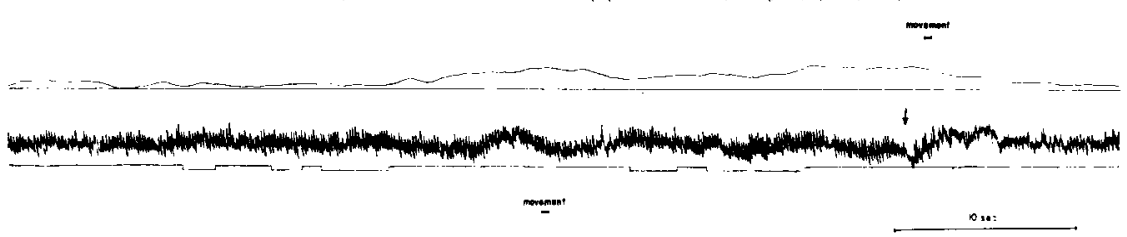

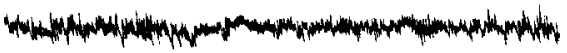

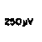

B $1000 \mathrm{CPS}$

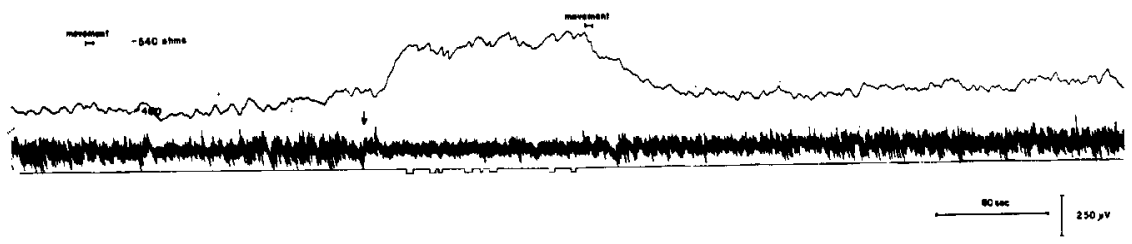

FIG. 8. Changes in the magnitude of impedance at $1000 \mathrm{cycle} / \mathrm{sec}$ in the subicular area during paradoxical sleep. This is raw data. A: A continuous record at fast speed. The top two lines are the impedance and a baseline. The next line is the spontaneous electrical activity. On the middle section a downward deflection of the bottom line indicates twitching of vibrissae. (This line does not appear in the other sections in which there was no twitching of vibrissae.) The arrows indicate the beginning and the end of the theta rhythm. B: The same presentation as each run of Fig. 6 . Unless otherwise stated, the rat is lying down quietly with his head down and his eyes closed.

abrupt termination of paradoxical sleep was the most striking temporal correlation of all.

At $32 \mathrm{cycle} / \mathrm{sec}$, the frequency response of the system is so slow and the spontaneous variation so large that these temporal correlations of the impedance with paradoxical sleep cannot be made more accurate than within about $20 \mathrm{sec}$. However, with this limitation, the same temporal correlations seen at higher frequencies hold.

In two animals, increases of impedance similar to those occurring before 
episodes of paradoxical sleep were frequently seen in isolation without the subsequent episode of paradoxical sleep (Fig. 9A, B). As in Fig. 9B, they frequently occurred at regular intervals. They almost invariably ended with a motion of the entire body, and low voltage fast EEG. They always occurred while an animal was in slow-wave sleep and had been in slow-wave sleep for a long enough time so that an episode of paradoxical sleep was ex-

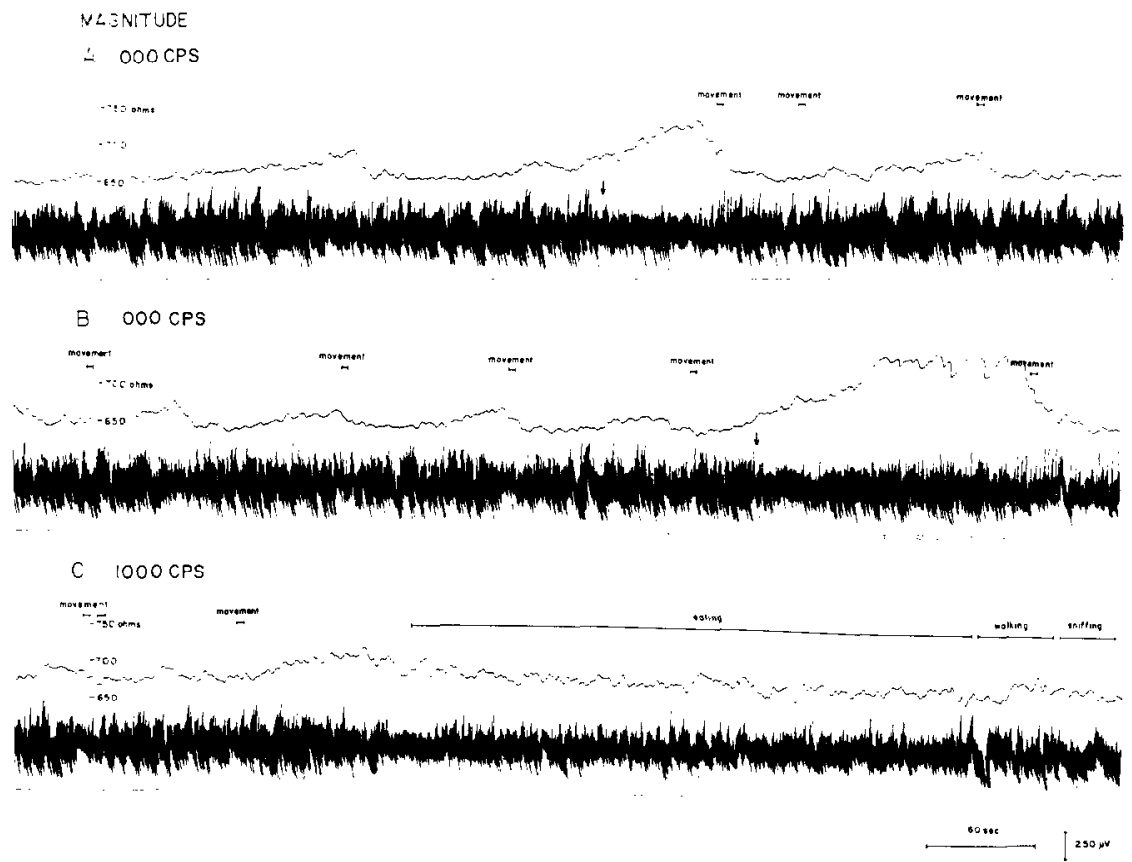

Fif. 9. Changes in the magnitude of impedance at $1,000 \mathrm{cycles} / \mathrm{sec}$ in the subicular area. The same rat as in Fig. 6. The same presentation as in each run of Fig. 6. A: A 50 -second episode of paradoxical sleep and two small increases. B: Four small increases of impedance occurring rhythmically before an episode of paradoxical sleep. C: Impedance changes during activity. Unless otherwise stated, the rat is lying down quietly with his head down and his eyes closed.

pected (14). These changes were sufficiently small and uncorrelated with other activity, so that it is impossible to say whether there were also changes in phase angle or at other frequencies. It would be necessary to measure these changes simultaneously in order to be sure.

A theta rhythm also occurs in the subicular area during arousal, yet there were no changes in impedance associated with the theta rhythm of arousal. During other types of muscular activity of the head (eating, munching, sniffing, and drinking), there was no consistent change in impedance in the subicular area. So neither a theta rhythm nor muscular activity of the head 
is the basis for the increased magnitude of impedance in the subiculum during paradoxical sleep.

Electrodes were in Ammon's horn of two rats, and in the fascia dentata and its hilus in one rat. In one animal with electrodes in Ammon's horn, there was a $1-2 \%$ increase in magnitude of impedance at 32 and 1,000 cycle/ sec associated with paradoxical sleep. No changes were seen in the other animals. Phase angle was not measured.

Those rats with electrodes in Ammon's horn, fascia dentata, or subicular area also were watched for hours while awake, eating, drinking, walking, sniffing-the repertoire of spontaneous behavior of a caged rat. No consistent correlation with impedance at any frequency was observed except during paradoxical sleep. However, during activity, the spontaneous variation in the magnitude of impedance often increased, so there may be correlations of impedance with some types of activity which were not appreciated (Fig. 9C).

The three rats with electrodes in Ammon's horn or fascia dentata were among the first to be run in this series, when loose electrodes and motion artifacts were especial problems. Therefore, this study cannot claim to give any data on relation of activity to impedance in Ammon's horn and fascia dentata.

\section{Analysis}

The major result can then be summarized. In the subicular area, associated with episodes of paradoxical sleep, there is an increase in magnitude of impedance of up to $25 \%$ which is constant in recurrent episodes and which is the same at 32 to $10,000 \mathrm{cycle} / \mathrm{sec}$. There are changes in the phase angle of up to $2 \mathrm{deg}$ more negative at 100 and $1,000 \mathrm{cycle} / \mathrm{sec}$.

Let us now go through possible mechanisms responsible for changes of impedance and determine which fit these data.

a. Changes in blood volume of the subicular area may well occur, and would be expected to increase the magnitude of the impedance equally at all frequencies, with no associated changes in phase angle. However, this contribution to the current flow is so small that it could not possibly cause the large changes seen, although changes in blood volume might be a small contribution. Specifically, since the blood volume of rat hippocampus is about $1 \%(7)$ and the specific resistance is $250 \mathrm{ohm}-\mathrm{cm}$, then if the entire blood volume were removed, the increase would be less than $2 \%$.

b. In previous papers, it has been argued that some current flows through cell processes in brain $(11,12)$, and this has indeed been shown to be the case by Ranck and BeMent (13) and Nicholson (8) in white matter. Therefore, changes in membrane resistance of cells could affect the impedance of brain. However, at high frequencies, the membrane resistance would have little effect on impedance of the brain, as the membrane resistance would be largely shorted out by the membrane capacity. Therefore, if impedance 
changes are at least partially due to changes in membrane resistance, the changes in magnitude of impedance should be greater at low frequencies rather than at high. There will also be large changes in phase angle at intermediate frequencies. ${ }^{3}$

Just what frequencies are "low" or "high" has been discussed in detail in an earlier paper (11), but, in general, a frequency (in cycle/sec) is low if it is less than about a tenth the reciprocal of the time constant of the membrane, and high if it is greater than ten times the reciprocal of this time constant. The time constants of cells in the subicular area is not known. Since the largest phase angles seen were at 32 or $100 \mathrm{cycle} / \mathrm{sec}$, a large population of cells has a time constant of the order of $\frac{1}{32}$ or $1 / 100$ of a second (presumably this population includes at least many neurons). The membrane resistance of this population cannot be contributing to the impedance changes.

The highest frequency used was $10,000 \mathrm{cycle} / \mathrm{sec}$. This is a low frequency for cell membranes with time constants of less than about $10^{-5} \mathrm{sec}$. Since specific membrane capacity is about the same in all cells, this is equivalent to about $10 \mathrm{ohm} \mathrm{cm}^{2}$. Therefore, it is conceivable that the impedance change could be due to some population of cells increasing its membrane resistance from a very low value up to about $10 \mathrm{ohm} \mathrm{cm}^{2}$. However, these cells would still be so leaky that increasing their membrane resistance would produce a very small increase in magnitude of tissue impedance, unless these cells had very short processes and were very numerous. I consider this possibility very unlikely, but cannot rule it out.

c. Increases in cytoplasmic resistance due to increased cytoplasmic resistivity or decreased cytoplasmic volume will affect impedance differently, depending on the membranc resistancc of the cell. If the membrane resistance is sufficiently low, so that much current flows through the fibers Iongitudinally, even at low frequencies, then the changes in amplitude of impedance will be about the same at all frequencies. If the membrane resistance of a cell is high, so that little current flows through the cell at low frequencies, then the impedance change due to increased cytoplasmic resistance will be much greater at high frequencics than at low frequencies. There would be small increases in phase angle of impedance in both cases. The impedance changes in the subicular area during paradoxical sleep are thus consistent with an increased cytoplasmic resistancc. However, if this factor alone were the basis of the tissue impedance change, it would imply an increase of cytoplasmic resistance of up to $20 \%$, if it were assumed that all the current flows through cytoplasm. Progressively larger increases would be implied for

${ }^{3}$ Just such reasoning has been used in an earlier paper on analyzing impedance changes during spreading cortical depression of Leao, where indeed there are contributions to impedance changes from changes in cell membrane resistance (12). 
assumptions of greater amounts of current flow through interstitial space. Changes of this magnitude are not known to occur. This factor cannot be eliminated, but it seems extremely unlikely.

d. Low resistance electrical connections between cells are not known to occur in the mammalian central nervous system, although many examples are known elsewhere. If they occur in the subicular area, then increasing this resistance between cells would have an effect on tissue impedance similar to that of increasing cytoplasm resistance. To produce the size of change seen in the subiculum, there would have to be a very extensive interconncction between cells. There is no reason to suspect that this is true, but, in view of our fragmentary knowledge of electrical connections between cells and of the subicular area, this possibility cannot be rejected completely.

e. Increases in the resistance to current flow through the interstitial space would cause a slightly greater increase in the magnitude of tissue impedance at low than at high frequencies (but too small a difference to be measured by these methods), and there would be no increase or a small increase in phase angle at high frequencies (12, pp. 8 and 12). This is just what is seen experimentally. This increased resistance could be due to: a decreased size of interstitial space, an increased tortuosity of the current path through the interstitial space due to frequent obstructions from formation of nexuses between cells, an increased resistivity of the interstitial space, or some combination of these. Impedance methods do not permit these possibilities to be distinguished. Changes in the interstitial space seem by far the most likely mechanism of the impedance change, for reasons to be given in the discussion.

Some combination of some or all the factors listed above might contribute to the explanation of the impedance changes seen. There are many views on the nature of interstitial space, neuroglia cells, and pathways of current flow in the brain. I have tried to make the possibilities listed above include all of these views. I can think of no other serious possibilities than those listed above.

\section{Discussion}

Most of the advantages and peculiarities of the method are discussed in the methods section. The inability to obtain stable magnitudes of impedance in all animals over periods of 1 hour or more is a weakness of the method at the present time. The increase in impedance with paradoxical sleep reported in this paper was found, invariably, in all animals, regardless of the stability of the impedance, and it involved periods of just minutes. Drifts in baseline occurred over much longer periods of time, so there seems to be no reason to question the method for the data reported. Some of the causes 
of the limitations of the method are known so that some of the limitations may well be removed in the future.

At present, magnitude and phase angle and each frequency must be measured separately. However, because of the constant changes in paradoxical sleep, separate measurements from different episodes of paradoxical sleep can be directly compared. If changes during a process which was not as "all or none" as paradoxical sleep were studied, this comparison would be more difficult, for instance, as in evaluating the small increases in impedance before an episode of paradoxical sleep. However, the method could easily be extended to measure magnitude and phase angle of impedance at more than one frequency simultaneously. If this had been done on the changes during paradoxical sleep in the subiculum, even more quantitative arguments could have been made about the possible mechanisms involved.

The more complete data collected by this method allow more clear interpretation than other studies on impcdance changes in chronic animals. There is not a unique interpretation in this case, but the alternatives can be delineated clearly enough so that other experimental methods may in the future be used to decide among them.

This change in magnitude of impedance of up to $25 \%$ is the largest ever reported in normal situations. Increases in spreading depression, in hypothermia and in cardiac arrest, of up to $80 \%$ have been reported $(3,12)$. These are the largest, but this change with paradoxical sleep is the next largest.

In the analysis section, several mechanisms which might contribute to the impedance change are given. Other types of experiments are necessary to distinguish these possibilities. However, it seems that by far the most likely basis for change is an increase in resistance of interstitial space. Changes of a magnitude sufficient to explain the results are known to occur in the brain.

Let us define an "equivalent conductive interstitial space" as the volume of interstitial space which has the same conductance as one filled with a fluid with a conductivity of $60 \mathrm{ohm}-\mathrm{cm}$ (the conductivity of cerebrospinal fluid) and a tortuosity factor of 1.42 (the increase in path length of all fibers are randomly oriented) (11, p. 157). The resistivity of the subicular area is of the order of $250 \mathrm{ohm}-\mathrm{cm}$; therefore, a $20 \%$ increase in magnitude of impedance would be equivalent to eliminating an equivalent conductive interstitial space of about $6.8 \%$. In spreading depression of Leao of the neocortex, an equivalent conductive interstitial space of about $10 \%$ is eliminated, as determined by impedance data similar to that of this study (12). The electron microscopic observations of Van Harreveld, Crowell and Malhotra (15) indicate a decrease in the size of the interstitial space after asphyxiation. The interstitial space of caudate, as measured by ventriculocysternal perfusion with inulin, decreases from $7-14$ to $1-4.5 \%$ with death (9). The size 
of the interstitial space of the brain seen in electron microscopy is a function of method of preparation of the tissue $(6,15)$.

The change in impedance in the subicular area is not spreading depression, since so much spontaneous electrical activity is present. However, in the rat, spreading depression initiated in neocortex goes throughout neocortex and paleocortex and goes up to or into subicular area, but does not pass into hippocampus. A spreading depression initiated in hippocampus spreads throughout the hippocampus, but does not cross subicular area into adjacent entorhinal cortex (5). Thus, in both spreading depression and in paradoxical sleep, there are suggestions of peculiarities of the interstitial space of the subicular areas.

The frequency of nexuses seen in the smooth muscle and heart is a function of prior experimental handling of the tissue (4). The amount of membrane surface fused as nexuses in mammalian central nervous system is a function of fixation (6). It is conceivable this same lability may occur normally.

No case of an increase in the resistivity of fluid in interstitial space is known. Changes in the ions in the interstitial space could not occur in a sufficient amount to cause the impedance changes seen. It is conceivable that a change in configuration of protein, mucopolysaccharide, or other molecules on the surface of cell membranes could affect the mobility of the ions.

It would be surprising if each of the possibilities for the mechanism responsible for the change in impedance were not associated with major changes in the general mode of cellular functioning in the subicular area, a change not only in rates of firing of neurons, but a global change in the nature of the tissue and interaction of cells. In paradoxical sleep, the nervous system seems to be operating almost in another mode. It is unprofitable to guess any further as to the relation of this impedance change to paradoxical sleep until it is known which mechanism is responsible. Interpretation is further hindered since remarkably little is known about the subicular area.

Adey, Kado and Walter (3) noted $0.3 \%$ increases in the magnitude of impedance at $1,000 \mathrm{cycle} / \mathrm{sec}$ in the amygdala of cats during paradoxical sleep. It is possible that they were observing the effect of the change in the subicular area nearby, or perhaps other parts of the brain also have similar changes to that in subicular area, or an entirely different process is going on. The small changes in the hippocampus also might be due to these same reasons.

Impedance changes in Ammon's horn associated with arousal, sensory input, pain, and anesthesia by Nembutal have been reported (1-3). As stated in the results sections, our results on Ammon's horn during activity are not good enough to warrant comparison. 


\section{References}

1. Adey, W. R., R. T. Kado, and J. Didio. 1962. Impedance measurements in brain tissue of animals using microvolt signals. Exptl. Neurol. 5: 47-66.

2. Adey, W. R., R. T. Kado, J. Didio, and W. J. Schindler. 1963. Impedance changes in cerebral tissue accompanying a learned discriminative performance in the cat. Exptl. Neurol. 7: 259-281.

3. AdEy, W. R., R. T. Kado, and D. O. Walter. 1965. Impedance characteristics of cortical and subcortical structures: evaluation of regional specificity in hypercapnea and hypothermia. Exptl. Neurol. 11: 190-216.

4. Barr, L., M. M. Dewey, and W. Berger. 1965. Propagation of action potentials and the structure of the nexus in cardiac muscle. J. Gen. Physiol. 48: 79-823.

5. Fifkova, E. 1964. EEG depression in the neo-, paleo-, and archicortical structures of the brain of the rat. Physiol. Bohemoslov. 13: 1-15.

6. Karlsson, V., and R. L. Shultz. 1965. Fixation of the central nervous system for electron microscopy by aldehyde perfusion I. J. Ultrastruct. Res. 12: 160-186.

7. NAIR, V., D. PALM, and L. J. Roth. 1960. Relative vascularity of certain anatomical arcas of the brain and other organs of the rat. Nature 188: 497-498.

8. Nicholson, P. W. 1965. Specific impedance of cerebral white matter. Exptl. Neurol. 13: $386-401$.

9. Rall, D. P., W. W. Oppelt, and C. S. Patlak. 1962. Extracellular space of brain as determined by diffusion of inulin from the ventricular system. Life Sci. No. 2, 43-48.

10. Ranck, J. B., JR. 1963. Specific impedance of rabbit cerebral cortex. Exptl. Neurol. 7: 144-152.

11. RancK, J. B., JR. 1963. Analysis of specific impedance of rabbit cerebral cortex. Exptl. Neurol. 7: 153-174.

12. Ranck, J. B., JR. 1964. Specific impedance of cerebral cortex during spreading depression, and an analysis of neuronal, neuroglial, and interstitial contributions. Exptl. Neurol. 9: 1-16.

13. Ranck, J. B., JR, and S. L. Be Ment. 1965. The specific impedance of the dorsal columns of cat: an anisotropic medium. Exptl. Neurol. 11: 451-463.

14. Roldan, E., T. Weiss, and E. Fifkora. 1963. Excitability changes during sleep cycle of the rat. Electroencephalog. Clin. Neurophysiol. 15: 775-785.

15. Van Harrevelo, A., J. Crowelt, and S. K. Malhotra. 1965. A study of extracellular space in central nervous tissue by frecze-substitution. J. Cell Biol. 25: $117-137$. 